\title{
The Value of Cervical Node Features in Predicting Long-Term Survival of Nasopharyngeal Carcinoma in the Intensity-Modulated Radiotherapy Era
}

\author{
Yun-Ming Tian ' \\ Lei Zeng ${ }^{2}$ \\ Yu-Hong Lan' \\ Xia Yuan ${ }^{3}$ \\ Li Bai ${ }^{1, *}$ \\ Fei $\operatorname{Han}^{4, *}$ \\ 'Department of Radiation Oncology, \\ Huizhou Central People's Hospital, \\ Huizhou, Guangdong, People's Republic \\ of China; ${ }^{2}$ Department of Medical \\ Oncology, The Second Affiliated Hospital \\ of Nanchang University, Nanchang, \\ Jiangxi, People's Republic of China; \\ ${ }^{3}$ Department of Medical Oncology, \\ Huizhou Central People's Hospital, \\ Huizhou, Guangdong, People's Republic \\ of China; ${ }^{4}$ Department of Radiation \\ Oncology, Sun Yat-Sen University Cancer \\ Centre; State Key Laboratory of \\ Oncology in South China; Collaborative \\ Innovation Centre for Cancer Medicine, \\ Guangdong, People's Republic of China \\ *These authors contributed equally to \\ this work
}

Objective: To investigate the prognostic value of cervical node features in patients with nasopharyngeal carcinoma (NPC) treated with intensity-modulated radiotherapy (IMRT) and build a prognostic nomogram to predict the long-term survival.

Methods: In this study, 1752 patients after IMRT from 2008 to 2011 were recruited. The clinical and laboratory characteristics and the nodal features including the nodal number, maximum dimension diameter, extranodal extension (ENE), and cervical node necrosis $(\mathrm{CNN})$ were retrospective analyzed. Univariate Cox and multivariate proportional hazard regression models were used to test the prognostic value of nodal features. Prognostic nomograms were established to predict survival.

Results: The 10-year distant metastases-free survival (DMFS) and disease-specific survival (DSS) rates were $86.5 \%$ and $80.8 \%$, respectively. Multivariate analysis showed that age, sex, lactate dehydrogenase (LDH), CNN, ENE, T stage, and N stage were independent factors for DSS. Two nomograms - nomogram A (without nodal features) and nomogram B (with nodal features) - were built. The calibration curve for the probability of DSS showed good agreement between prediction by nomogram and the actual observation. The C-index of nomogram $\mathrm{B}$ was higher than that for nomogram A in predicting DSS $(0.708$ vs $0.676, \mathrm{P}<0.01)$. Conclusion: The nodal features including ENE and CNN were negative prognostic factors for NPC, and the prognostic nomogram incorporating the nodal features was more accurate in predicting survival than the nomogram without nodal features.

Keywords: nodal features, extranodal extension, nodal necrosis, nasopharyngeal carcinoma, prognostic value

\section{Introduction}

Nasopharyngeal carcinoma (NPC) is a unique head and neck malignancy with a distinct pattern of geographical distribution, which is commonly seen in Southeast Asians. ${ }^{1,2}$ Because the nasopharynx has an abundant lymphatic drainage system, 80-90\% patients with NPC show cervical node metastasis as detected by magnetic resonance imaging (MRI) or computed tomography (CT). ${ }^{3,4}$ Furthermore, cervical node metastasis is one of the most valuable prognostic factors to predict the high rate of distant metastasis and poor survival and is a critical component of the TNM staging system. ${ }^{5}$ However, in the NPC staging system, the $\mathrm{N}$-classification is mainly determined by size, level, and laterality of the cervical node, while the other potential valuable features are not included.
Department of Radiation Oncology, Hui Zhou Municipal Central Hospital, No. 4I Eling North Road, Huizhou, Guangdong, People's Republic of China

Email bailihuizhou@।26.com

Fei Han

Department of Radiation Oncology, Sun Yat-Sen University Cancer Center,

No. 65I Dongfeng East Road, Guangzhou, People's Republic of China

Email hanfeisysucc@126.com 
Accurate and comprehensive assessment of the metastatic cervical node is helpful to predict prognosis and evaluate individual treatment options in NPC patients. Recent studies have suggested that nodal features including extranodal extension (ENE) and number of nodes are strong prognostic factors of poor outcome in patients with head and neck squamous cell carcinoma (HNSCC) and were included in the 8th edition of American Joint Committee on Cancer (AJCC) staging system. ${ }^{6-9}$ However, the value of these nodal features in predicting long-term survival in patients with NPC in the intensity-modulated radiation therapy (IMRT) era is relatively limited, and some of the published results are inconsistent. ${ }^{10-13}$

This study was conducted to evaluate the prognostic value of the radiologic features of metastatic neck nodes in IMRT-treated NPC patients. A prognostic nomogram with the radiologic features was built to select patients with a high risk of treatment failure.

\section{Methods and Materials Materials}

We retrospectively reviewed patients with non-metastatic NPC between January 2008 and December 2011, and the inclusion criteria were as follows: (1) pathologically proven NPC, (2) cervical lymph node metastases $(\mathrm{N}+)$, (3) complete imaging and clinical data, and (4) full-course IMRT. Eventually, 1753 NPC patients were enrolled in this study. All patients underwent contrast-enhanced MRI of the nasopharynx and neck for the staging evaluation; $\mathrm{CT}$ and singlephoton emission CT (SPECT) were used to exclude distant metastasis. Positron emission tomography (PET-CT) was also recommended when clinically indicated. Patients were re-staged according to the 7th edition AJCC staging system.

The study was conducted in accordance with the tenets of the Helsinki declaration and was approved by the institutional ethics committee of Sun Yat-sen University Cancer Center. Written informed consent was obtained from all participants. All protocols were performed in accordance with the relevant guidelines and regulations.

\section{MRI Image Acquisition}

MRI was performed with a $1.5-\mathrm{T}$ or 3.0-T system (SignaCV/i, General Electric Healthcare) in all patients before treatment. The scanning area ranged from the suprasellar cistern to the superior border of the thoracic cage. Fast spin-echo (FSE) T1-weighted images (T1WI) on the axial, sagittal, and coronal planes and axial FSE T2- weighted images (T2WI) were obtained before injection of contrast material. After intravenous injection of gadopentetate dimeglumine (Gd-DTPA, $0.1 \mathrm{mmol} / \mathrm{kg}$ body weight), axial and sagittal T1-weighted spin-echo and coronal T1-weighted fat-suppressed spin-echo images were sequentially obtained. The parameters for the T1WI and T2WI scans were TR $=500-600 \mathrm{~ms}$ and $\mathrm{TE}=10-20 \mathrm{~ms}$, and $\mathrm{TR}=4000-6000 \mathrm{~ms}$ and $\mathrm{TE}=95-110 \mathrm{~ms}$, respectively. The thickness of the MRI layers was $5 \mathrm{~mm}$ for the axial sequences and $2 \mathrm{~mm}$ for the coronal or sagittal sequences, with a 1-mm intersection gap.

\section{MRI Assessment for Metastatic Neck Nodes \\ Metastatic Neck Nodes}

The MRI was interpreted by two radiation oncologists specialized in NPC with more than 10 years' experience. Any disagreements were resolved by consensus with the team. The main MRI-based diagnostic criteria for the metastatic neck nodes were (1) retropharyngeal lymph nodes with a minimal axial diameter of $5 \mathrm{~mm}$ and other cervical lymph nodes with a minimal axial diameter of $10 \mathrm{~mm}$; (2) groups of $\geq 3$ lymph nodes in the same area with a minimal axial diameter of $8 \mathrm{~mm}$; and (3) lymph nodes of any size with ENE or central necrosis or enhancing rim. ${ }^{14,15}$ Besides the $\mathrm{N}$ classification, other radiologic features including the maximal diameter, number, ENE, and nodal necrosis were recorded.

\section{Cervical Nodal Necrosis (CNN)}

The criteria for $\mathrm{CNN}$ were a focal area of high signal intensity on T2WI or low signal intensity on T1WI and non-enhancement on contrast-enhanced T1WI (Figure 1).

\section{Radiologic Extranodal Extension ( $r E N E$ )}

The criteria for rENE on MRI were an unequivocal illdefined border, mainly including the loss of sharp plane between the nodal capsule and the surrounding fat and the infiltration into surrounding structures (Figure 1).

\section{Radiotherapy and Chemotherapy}

All patients were treated with the full course of IMRT. The target volume included gross tumor volume in the nasopharynx (GTV-nx) and in the involved cervical lymph nodes (GTV-nd) and the high- and low-risk clinical target volume (CTV1 and CTV2, respectively). A detailed description of IMRT including the delineation and prescription dose has been previously reported. ${ }^{16}$ 


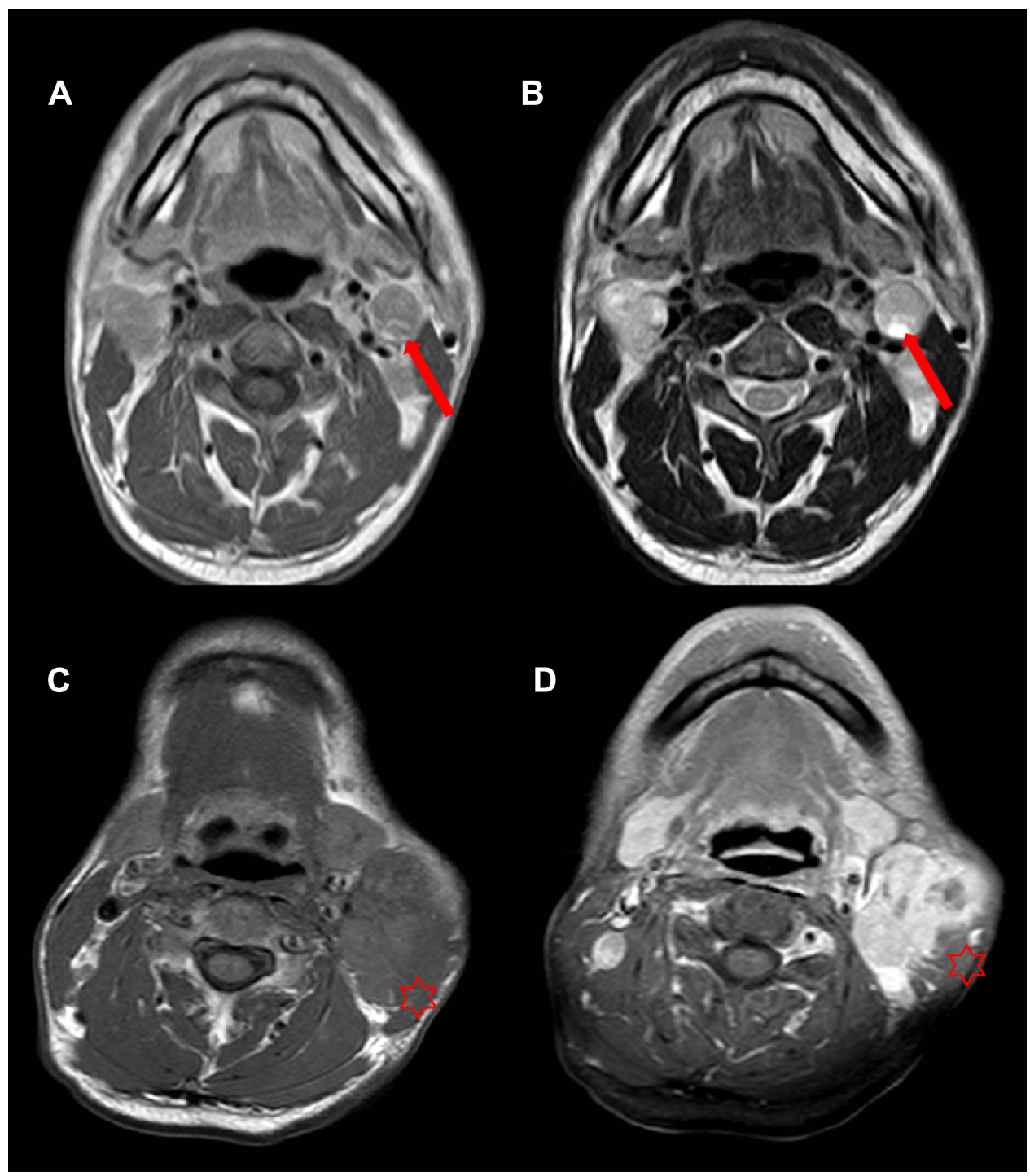

Figure I Representative MRI results illustrating the cervical nodal necrosis (CNN) and extranodal extension (ENE) in two patients with NPC. (A) Axial TIWI and (B) Axial T2WI in a 4I-year-old man with CNN (arrows); (C) Axial TIWI and (D) contrast-enhanced TIWI in a 45-year-old man with ENE infiltrating the adjacent muscle (stars).

According to the institutional guidelines in the center, concurrent chemoradiotherapy (CCRT) was the main treatment for stage II-IVa/b disease; induction chemotherapy (IC) or adjuvant chemotherapy (AC) was also administrated in patients with more advanced or bulky disease. Of the 1752 patients, 260 were treated with radiotherapy alone and 1492 patients received a combination of chemoradiotherapy modalities including CCRT alone ( $\mathrm{n}=647), \mathrm{IC}+\mathrm{CCRT}(\mathrm{n}=478)$, IC alone $(\mathrm{n}=271), \mathrm{CCRT}+\mathrm{AC}(\mathrm{n}=65)$, and other modalities of chemotherapy $(\mathrm{n}=31)$.

\section{Follow-Up and Statistical Analysis}

After the completion of treatment, patients were followedup every 3 months during the first 2 years, every 6 months in 3-5 years, and yearly thereafter until death.

All statistical analyses were performed using SPSS software (version 19, IBM Corporation, Armonk, NY USA). ROC curve was performed to determine the cutoff point for the continuous variables including age, maximal diameter and number of node. The Kaplan-Meier method was used to calculate the rates of local recurrencefree survival (LRFS), regional recurrence-free survival 
(RRFS), distant metastasis-free survival (DMFS), progression-free survival (PFS), overall survival (OS), and disease-specific survival (DSS). The Log rank test was used to compare the survival curves. Multivariate analysis with Cox proportional hazards model was performed to test for independent prognostic factors. All statistical tests were two-sided, and $\mathrm{P}<0.05$ was considered to indicate statistical significance.

The nomogram was built based on the results of multivariate analysis using the package of RMS in R version 3.6.1. The final prediction model was built using a backward step-down selection process with the Akaike information criterion. The performance of the nomogram was evaluated by concordance index (C-index) and assessed by comparing nomogram-predicted versus observed Kaplan-Meier estimates of survival probability. $^{17,18}$ A comparison between nomogram A (without ENE or CNN) and nomogram B (with ENE and $\mathrm{CNN}$ ) was performed with the rcorr.cens function in the $\mathrm{R}$ Hmisc package and evaluated by the $\mathrm{C}$-index. The accuracy of prognosis prediction was evaluated by the $\mathrm{C}$-index value.

\section{Results}

\section{Patient Characteristics}

The median age for the cohort was 44 years (range, 13-76 years), with a male:female ratio of $2.7: 1$. The median maximal axial diameter was $19 \mathrm{~mm}$ (range, 5-89 $\mathrm{mm}$ ). The number of patients with N1, N2, and N3 were 837, 597, and 315, respectively. Furthermore, 768 patients had $\mathrm{CNN}$, and 825 patients had ENE. The patient details are presented in Table 1.

\section{Survival and Pattern of Failure}

The median follow-up for the whole cohort was 124 months, and 72 patients (4\%) were lost to follow-up after 3-91 months. By the last follow-up date, distant metastasis occurred in 245 patients $(13.9 \%)$ and was the most common pattern of failure. Among patients with distant metastasis, 156 had single-organ metastasis and 65 had multipleorgan metastasis. The bone was the most common metastatic site, followed by the lung and the liver. The 10 -year DMFS rate was $86.5 \%$.

A total of 161 patients developed local and/or regional failure; of these, 119, 38, and four patients had local failure only, regional failure only, and both, respectively. The 10-year LRFS, RRFS, and PFS rates were 92.6\%, 97.4\%, and $78.6 \%$, respectively.

A total of 347 patients died. Among these, 188 patients (188/347, 54.2\%) died from distant metastasis, 104 (104/ $347,29.9 \%$ ) died from the local and/or regional failure, 14 $(14 / 347,4.0 \%)$ died from radiation-related complications, $18(18 / 347,5.2 \%)$ died from other malignant tumors, eight $(8 / 347,2.3 \%)$ died from the internal medication, and 15 $(15 / 347,4.3 \%)$ died of unknown causes. The 10 -year OS and DSS rates were $79.8 \%$ and $80.8 \%$. The details are presented in Table 2 .

\section{Univariate and Multivariate Analysis}

The results of univariate analysis showed that $\mathrm{LDH}$, nodal number, $\mathrm{CNN}$, rENE, $\mathrm{T}$ stage, and clinical stage were identified as significant prognostic factors for LRFS, DMFS, PFS, and DSS, while N stage was also identified as a significant prognostic factor for DMFS, PFS, and DSS (Table 1).

Consistent with the results of our univariate analysis, the multivariate analysis also showed that $\mathrm{CNN}$, rENE, T stage, and $\mathrm{N}$ stage were significant prognostic factors for DMFS, PFS, and DSS. However, the clinical stage and nodal number were not independent prognostic factors. The 10-year DSS, DMFS, and PFS for patients with CNN were poorer than those for patients without CNN, with $70.8 \%$ vs $88.6 \%, 80.8 \%$ vs $91.9 \%, 68.7 \%$ vs $86.2 \%$ (Figure 2). The 10-year DSS, DMFS, and PFS for patients with rENE were also significantly poorer than those for patients without rENE, with $71.7 \%$ vs $86.7 \%, 79.8 \%$ vs $90.1 \%, 70.1 \%$ vs $86.0 \%$ (Figure 3 ).

The factors of T stage, $\mathrm{LDH}$, and $\mathrm{CNN}$ were associated with LRFS, and the $\mathrm{N}$ stage and $\mathrm{CNN}$ were associated with RRFS. The details of the multivariate analysis are shown in Table 3.

\section{Establishment of Nomogram Models for DSS, with or Without Nodal Features}

First, we built a nomogram (Nomogram A) to predict the 5- and 10-year DSS rate only, based on independent prognostic factors of age, sex, $\mathrm{LDH}, \mathrm{T}$ stage, and $\mathrm{N}$ stage. Then, with the combinations of ENE and CNN, a new nomogram - nomogram B-was built to predict the 5- and 10 -year DSS rate. Each variable had a corresponding score according to the point scale, and the total score was achieved by calculating the score of each variable. Next, by mapping the total score on the probability scale, the 5- 
Table I Characteristics of 1752 Patients and Univariate Analysis

\begin{tabular}{|c|c|c|c|c|c|c|c|}
\hline Characteristics & No. & IOy-LRFS (\%) & $P$ value & IOy-DMFS (\%) & $P$ value & $10 y-D S S(\%)$ & $P$ value \\
\hline \multicolumn{8}{|l|}{ Gender, } \\
\hline Male & 1279 & 93.8 & 0.13 & 85.8 & 0.01 & 78.7 & $<0.01$ \\
\hline Female & 473 & 90.7 & & 91.7 & & 86.2 & \\
\hline \multicolumn{8}{|l|}{ Age (year) } \\
\hline$<55 y$ & 881 & 92.8 & 0.15 & 88.5 & 0.60 & 82.5 & 0.01 \\
\hline$\geq 55 y$ & 871 & 91.6 & & 86.8 & & 73.4 & \\
\hline \multicolumn{8}{|l|}{ LDH(year) } \\
\hline$\leq 245$ & 1634 & 92.0 & 0.02 & 88.3 & $<0.01$ & 82.0 & $<0.01$ \\
\hline$>245$ & 107 & 83.1 & & 74.5 & & 61.9 & \\
\hline \multicolumn{8}{|c|}{ Maximal diameter $(\mathrm{cm})$} \\
\hline$\leq 2.5$ & 1188 & 92.1 & 0.85 & 89.4 & $<0.01$ & 83.4 & $<0.01$ \\
\hline$>2.5$ & 564 & 91.6 & & 81.5 & & 72.8 & \\
\hline \multicolumn{8}{|l|}{ Number of node } \\
\hline$\leq 3$ & 937 & 93.4 & 0.03 & 91.3 & $<0.01$ & 86.0 & $<0.01$ \\
\hline$>3$ & 915 & 88.3 & & 80.0 & & 70.9 & \\
\hline \multicolumn{8}{|l|}{$\mathrm{CNN}$} \\
\hline Yes & 768 & 88.7 & 0.01 & 80.8 & $<0.01$ & 70.8 & $<0.01$ \\
\hline No & 984 & 93.6 & & 91.9 & & 88.6 & \\
\hline \multicolumn{8}{|l|}{ ENE } \\
\hline Yes & 825 & 89.4 & 0.02 & 79.8 & $<0.01$ & 71.7 & $<0.01$ \\
\hline No & 927 & 93.5 & & 90.1 & & 86.7 & \\
\hline \multicolumn{8}{|l|}{$\mathrm{T}$ stage } \\
\hline $\mathrm{TI}$ & 92 & 98.8 & $<0.01$ & 95.6 & $<0.01$ & 95.6 & $<0.01$ \\
\hline $\mathrm{T} 2$ & 430 & 93.9 & & 93.5 & & 87.4 & \\
\hline $\mathrm{T} 3$ & 872 & 93.7 & & 86.4 & & 80.8 & \\
\hline $\mathrm{T} 4$ & 358 & 81.3 & & 80.0 & & 68.4 & \\
\hline \multicolumn{8}{|l|}{$N$ stage } \\
\hline NI & 837 & 93.4 & 0.91 & 92.2 & $<0.01$ & 86.9 & $<0.01$ \\
\hline N2 & 597 & 92.5 & & 87.9 & & 81.0 & \\
\hline N3 & 315 & 91.4 & & 71.8 & & 63.7 & \\
\hline \multicolumn{8}{|l|}{ Clinical stage } \\
\hline ॥ & 264 & 94.9 & $<0.01$ & 96.4 & $<0.01$ & 93.0 & $<0.01$ \\
\hline III & 852 & 94.6 & & 90.5 & & 85.5 & \\
\hline $\mathrm{IVa}+\mathrm{b}$ & 836 & 85.7 & & 78.1 & & 69.1 & \\
\hline \multicolumn{8}{|l|}{ Chemotherapy } \\
\hline Yes & 1492 & 92.7 & 0.76 & 86.5 & $<0.01$ & 70.9 & 0.07 \\
\hline No & 260 & 91.3 & & 92.1 & & 79.5 & \\
\hline
\end{tabular}


Table 2 Failure Patterns in Nasopharyngeal Carcinoma (NPC) Patients

\begin{tabular}{|c|c|c|c|c|c|c|}
\hline Failure Pattern & ENE(+)(No.) & ENE(-)(No.) & $P$ value & CNN(+)(No.) & CNN(-)(No.) & $P$ value \\
\hline \multicolumn{7}{|l|}{ Local-regional failure } \\
\hline Local only & 66 & 53 & & 62 & 57 & \\
\hline Regional only & 30 & 8 & & 27 & 11 & \\
\hline Local+regional & 2 & 2 & & 3 & 1 & \\
\hline Total & 98 & 63 & 0.02 & 92 & 69 & 0.04 \\
\hline \multicolumn{7}{|l|}{ Distant metastasis } \\
\hline Bone only & 43 & 22 & & 53 & 12 & \\
\hline Lung only & 30 & 20 & & 31 & 19 & \\
\hline Liver only & 26 & 9 & & 22 & 13 & \\
\hline Other single organ & 5 & 3 & & 6 & 2 & \\
\hline Multiple organs & 47 & 16 & & 52 & 11 & \\
\hline Total & 151 & 70 & $<0.01$ & 164 & 57 & $<0.01$ \\
\hline \multicolumn{7}{|l|}{ Cause of death } \\
\hline Distant metastasis & 140 & 53 & & 136 & 57 & \\
\hline Local/regional failure & 55 & 44 & & 58 & $4 \mathrm{I}$ & \\
\hline Complications & 8 & 6 & & 10 & 4 & \\
\hline Other cancers & 10 & 8 & & 12 & 6 & \\
\hline Internal medication & 6 & 2 & & 7 & 1 & \\
\hline Unknown & 10 & 5 & & 12 & 3 & \\
\hline Total & 229 & 118 & $<0.01$ & 232 & 115 & $<0.01$ \\
\hline
\end{tabular}

and 10-year DSS rate probabilities could be estimated (Figure 4).

A calibration curve showed good agreement between prediction and observation in the probability of 5- and 10-year DSS. In Figure 5, the y-axes represent the observed DSS estimated by the Kaplan-Meier method, the X-axes represent the predicted DSS calculated by the nomogram, and the solid lines represent the ideal reference line for which predicted survival corresponds with the actual DSS. The C-index of nomogram B was 0.708 (95\% CI: 0.681-0.733), which was higher than the C-index of nomogram A $(0.676,95 \% \mathrm{CI}$ : 0.649-0.703). These results showed that nomogram $\mathrm{B}$ displayed better accuracy in predicting recurrence than nomogram A.

\section{Discussion}

With the common application of IMRT in patients with NPC, the high rate of local control and reduced complications has been reported in several studies. ${ }^{16,19}$ In our previous study, the 10 -year LRFS rate for patients treated
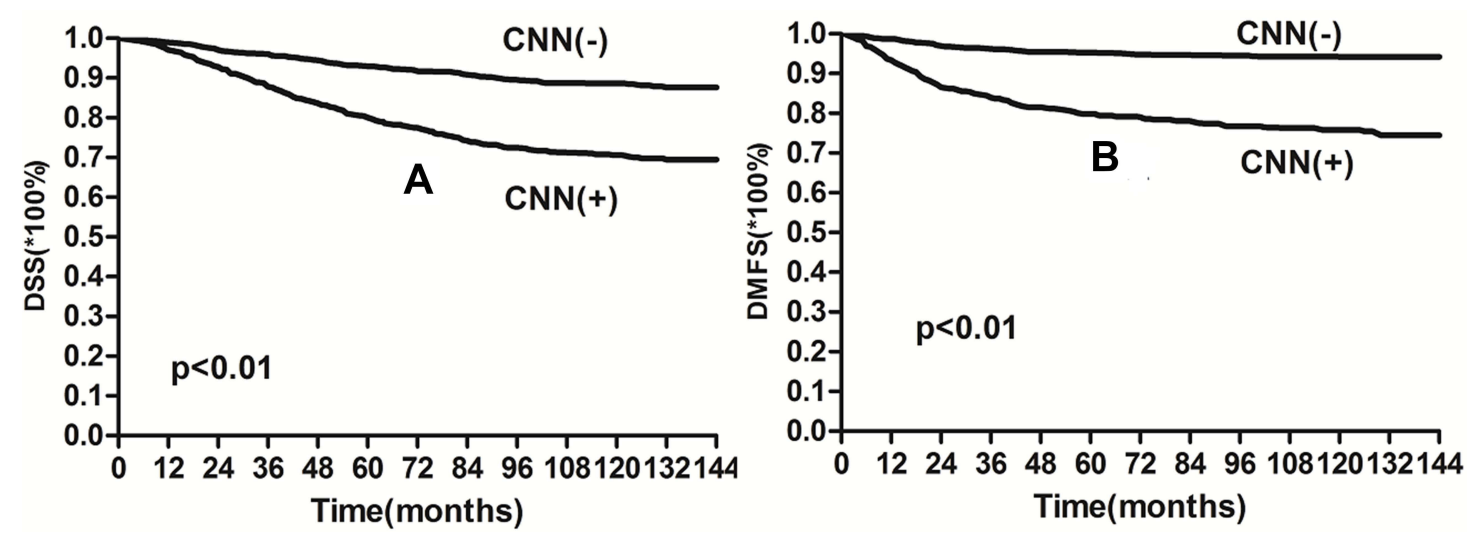

Figure 2 Kaplan-Meier curves showing (A) DSS and (B) DMFS for the CNN and non-CNN groups. 

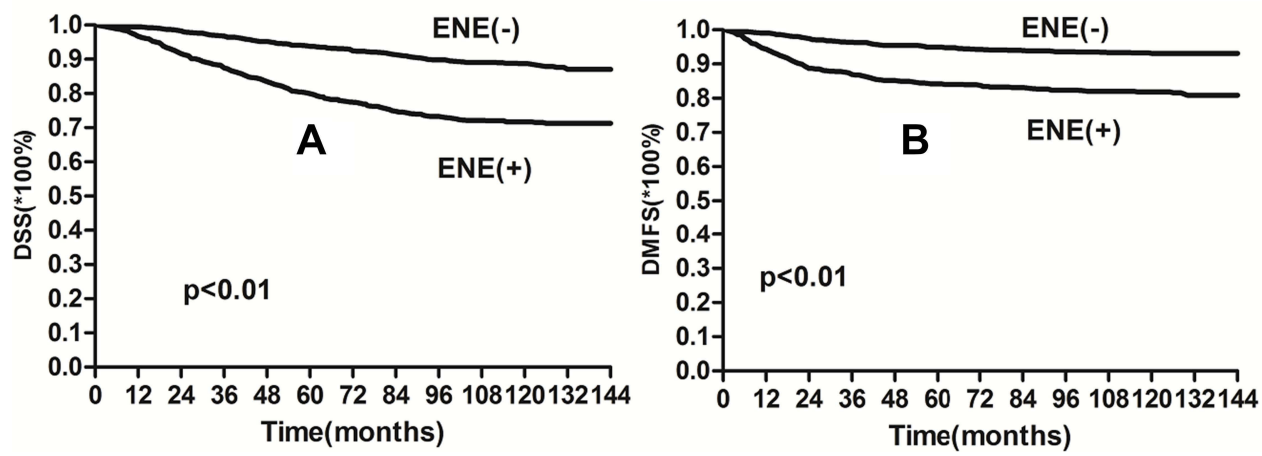

Figure 3 Kaplan-Meier curves showing (A) DSS and (B) DMFS for the ENE and non-ENE groups.

with IMRT was up to $92 \%$, significantly higher than those in the era of conventional RT. ${ }^{20}$ However, the incidence of distant metastasis was still $15-20 \%$ and becoming the most common cause of treatment failure. The neck nodal metastasis was shown to be the most important determinant of distant metastasis and poor survival. Therefore, the comprehensive evaluations of nodal morphological features might well help to select patients with high risk of treatment failure. However, the AJCC N-classification of nodal disease was only based on the metastasis size, laterality, and level; other nodal features associated with treatment failure were not evaluated. In the present study, we found that nodal features such as ENE and CNN were also associated with poor survival and high risk of distant metastasis. The nomogram that included the factors of nodal features showed better accuracy in predicting survival than the nomogram without nodal features.

Extranodal extension has been shown to be an overexpression of some factors that contribute to disease progression, such as matrix metalloproteinases and epidermal growth factor receptor gene $(E G F R) .{ }^{21}$ Extranodal extension has always been associated with a high incidence of distant metastasis, and it is therefore considered a critical parameter in upstaging the $\mathrm{N}$ classification in the new AJCC TNM stage system (8th edition, 2017) for nonviral-related head and neck cancers. ${ }^{10}$ Furthermore, ENE after surgery is an important indicator of intensive postoperative chemotherapy for patients with $\mathrm{HNSCC}$, given the high risk of distant metastasis. In the study related to 266 patients with tongue cancer, the 5-year OS rates were $75 \%, 50 \%$, and $30 \%$ for patients with $\mathrm{N}(-), \mathrm{N}(+)$ with ENE $(-)$, and $\mathrm{N}(+)$ with $\operatorname{ENE}(+)(\mathrm{p}<0.01)$. The incidence of distant metastasis for patients with $\mathrm{N}(+)$ with $\operatorname{ENE}(+)$ was up to $52 \% .^{7}$ In the study of 258 patients with HNSCC, the 3-year OS and DMFS rates for patients with $\mathrm{rENE}(+)$ were only $64.3 \%$ and $72.3 \%(\mathrm{p}<0.01)$, respectively, significantly lower than the rates of $82.8 \%$ and $90.6 \%$, respectively, in patients with $\operatorname{rENE}(-) .{ }^{6}$ For the surgery is not the primary treatment and no surgical specimen is available, the studies related to the prognostic value of ENE in patients with NPC were limited. As per evidence, CT and MRI exhibit similar accuracies in the diagnosis of ENE when compared with pathological assessment methods. ${ }^{22}$ Several studies related to NPC have shown that patients with ENE were associated with high risk of metastasis and poor outcome. ${ }^{10-13}$ In the report related to 1616 patients with NPC, the patients with $\mathrm{rENE}(+)$ had a significantly lower 5-year DMFS $(73.8 \%$ vs $88.4 \%, \mathrm{p}<0.01)$ and OS $(77.3 \%$ vs $87.6 \%$, $\mathrm{p}<0.01)$ than patients with $\mathrm{rENE}(-)$, and proposed to refine the N-classification according to the rENE. ${ }^{12}$ In a retrospective analysis of 1226 patients, Liu et al showed that $\mathrm{rENE}(+)$ was an independent prognostic factor for OS, but not for PFS and DMFS. ${ }^{13}$ Similar to Liu et al's report, we also found that ENE was a negative independent factor for DMFS and OS. ${ }^{13}$ Furthermore, recent studies have shown that the grade of ENE according to the extent can be more accurate to predict the survival. In a study by $\mathrm{Lu}$ et al, ${ }^{12}$ the $\mathrm{ENE}(+)$ was grading as three levels as G1, G2, and G3, and the results showed that grade G2 and G3 ENE were independent prognostic factors for distant metastasis and death in NPC. In another study reported by Ai et al, ${ }^{11}$ the $\mathrm{ENE}(+)$ was graded as follows: G1 (infiltration of surrounding fat) and G2 (infiltration of muscle/skin). It further showed that patients with G2 ENE were associated with significantly poorer RRFS, DMFS, and OS than those with G1 ENE.

Necrosis is another valuable morphological feature in the diagnosis of nodal metastasis and is common in NPC patients with an incidence of $22-42 \%{ }^{3}$ Previous studies 
Table 3 Multivariate Analysis of Prognostic Factors

\begin{tabular}{|c|c|c|c|}
\hline Variables & HR Value & $95 \% \mathrm{Cl}$ & $P$ value \\
\hline \multicolumn{4}{|l|}{ DSS } \\
\hline Age & $\mathrm{I} .47$ & $1.20-1.80$ & 0.01 \\
\hline Gender & 1.35 & $1.06-1.73$ & $<0.01$ \\
\hline LDH & 1.64 & I.19-2.24 & 0.01 \\
\hline CNN & 1.78 & 1.39-2.26 & $<0.01$ \\
\hline ENE & $\mathrm{I} .43$ & $1.10-1.85$ & $<0.01$ \\
\hline $\mathrm{T}$ stage & 1.81 & $1.53-2.08$ & 0.01 \\
\hline $\mathrm{N}$ stage & 1.42 & $1.23-1.63$ & $<0.01$ \\
\hline \multicolumn{4}{|l|}{ OS } \\
\hline Age & 1.62 & $1.33-1.97$ & $<0.01$ \\
\hline Gender & 1.35 & I.07-1.70 & 0.01 \\
\hline LDH & 1.58 & $1.16-2.15$ & 0.01 \\
\hline CNN & 1.64 & $1.31-2.07$ & $<0.01$ \\
\hline ENE & 1.33 & $1.05-1.70$ & $<0.01$ \\
\hline T stage & 1.78 & $1.56-2.04$ & 0.01 \\
\hline$N$ stage & $\mathrm{I} .4 \mathrm{I}$ & $1.24-1.62$ & $<0.01$ \\
\hline \multicolumn{4}{|l|}{ PFS } \\
\hline Gender & 1.52 & $1.17-1.96$ & 0.01 \\
\hline LDH & 1.58 & $1.45-2.05$ & 0.03 \\
\hline CNN & 1.71 & $1.33-2.17$ & $<0.01$ \\
\hline ENE & 1.39 & $\mid .07-1.81$ & $<0.01$ \\
\hline T stage & 1.60 & I.39-1.84 & 0.01 \\
\hline$N$ stage & 1.34 & $1.16-1.55$ & $<0.01$ \\
\hline \multicolumn{4}{|l|}{ DMFS } \\
\hline Gender & 1.60 & $1.13-2.26$ & 0.01 \\
\hline $\mathrm{T}$ stage & 1.72 & $1.42-2.08$ & $<0.01$ \\
\hline $\mathrm{N}$ stage & 1.62 & $1.34-1.96$ & 0.01 \\
\hline CNN & 1.81 & $1.29-2.55$ & $<0.01$ \\
\hline ENE & 1.60 & I.II-2.30 & $<0.01$ \\
\hline \multicolumn{4}{|l|}{ LRFS } \\
\hline $\mathrm{T}$ stage & 2.05 & $1.62-2.68$ & $<0.01$ \\
\hline LDH & 1.81 & $1.07-3.06$ & 0.01 \\
\hline CNN & 1.57 & $1.62-2.20$ & 0.01 \\
\hline \multicolumn{4}{|l|}{ RRFS } \\
\hline$N$ stage & 1.58 & $|| 2-2.5 \mid$. & $<0.01$ \\
\hline CNN & 4.20 & $2.09-8.45$ & $<0.01$ \\
\hline
\end{tabular}

have shown that tumor necrosis was associated with tumor hypoxia induced by the high tumor volume and rapid tumor growth. ${ }^{23}$ The presence of hypoxia in the tumor exhibits poor sensitivity to chemoradiotherapy and accelerates progression during treatment. Furthermore, studies have shown the association between the presence of $\mathrm{CNN}$ on $\mathrm{CT}$ and the poor survival after chemoradiotherapy in patients with head and neck cancer including NPC. Zoumalan et al reported that $\mathrm{CNN}$ at the preoperative CT was a useful indicator of ENE and an important negative prognostic indicator in patients with HNSCC. ${ }^{24}$ In Lan et al's ${ }^{25}$ retrospective analysis that included 1800 NPC patients the 5-year OS and DMFS rates of the $\mathrm{CNN}$ and non-CNN groups were $78.8 \%$ vs $91.8 \%$ and $78.4 \%$ vs $91.6 \%$, respectively. The distant metastasis rate in the CNN group was $18.7 \%$, significantly higher than that in the non-CNN group $(4.6 \%)$ $(p<0.01)$. In a study by Liu et al, CNN on MRI was also demonstrated as a significant negative prognostic factor for OS, LRRFS, and DMFS, and patients with CNN had significantly inferior 5-year OS $(82.6 \%$ vs $87.8 \%$ ), LRRFS (86\% vs $92.1 \%$ ), and DMFS ( $81.6 \%$ vs $89.5 \%$ ) than those without $\mathrm{CNN}{ }^{13}$ Similar to these results, the present study also indicated that nodal necrosis was associated with poor survival, and patients with CNN had a poorer 10 -year DSS (70.8\% vs $88.6 \%)$ and DMFS $(80.8 \%$ vs $91.9 \%)$ than those without CNN, respectively.

Series nomograms based on independent factors have been established to predict the survival in NPC patients and shown to be more accurate than the TNM staging systems, which is helpful for selection of patients with high risk of treatment failures. ${ }^{26,27}$ Such patients may benefit from the more aggressive therapy than others. For patients with high risk of local recurrence, neoadjuvant chemotherapy was recommended by reducing tumor volume and improving tumor control. ${ }^{27}$ Furthermore, adjuvant chemotherapy after radiotherapy may be administered for patients with high risk of distant metastasis. ${ }^{28}$ However, most nomograms do not include nodal features such as ENE and CNN. In our study, the nomogram that included ENE and CNN showed better accuracy in predicting the 5- and 10year DSS than the one that did not include these nodal features. The former nomogram may hence be more appropriate than the latter to stratify patients requiring individual treatment.

Our study has some limitations. First, this is a retrospective study related to the influence of nodal features on survival; therefore, some selection bias related to the treatment might have influenced the results. Second, for the diagnosis of ENE and CNN was based on the MRI, the diagnostic accuracy may be poorer when compared with the histology. Third, the prognostic nomogram was not externally validated and future external validation with larger cohorts from 

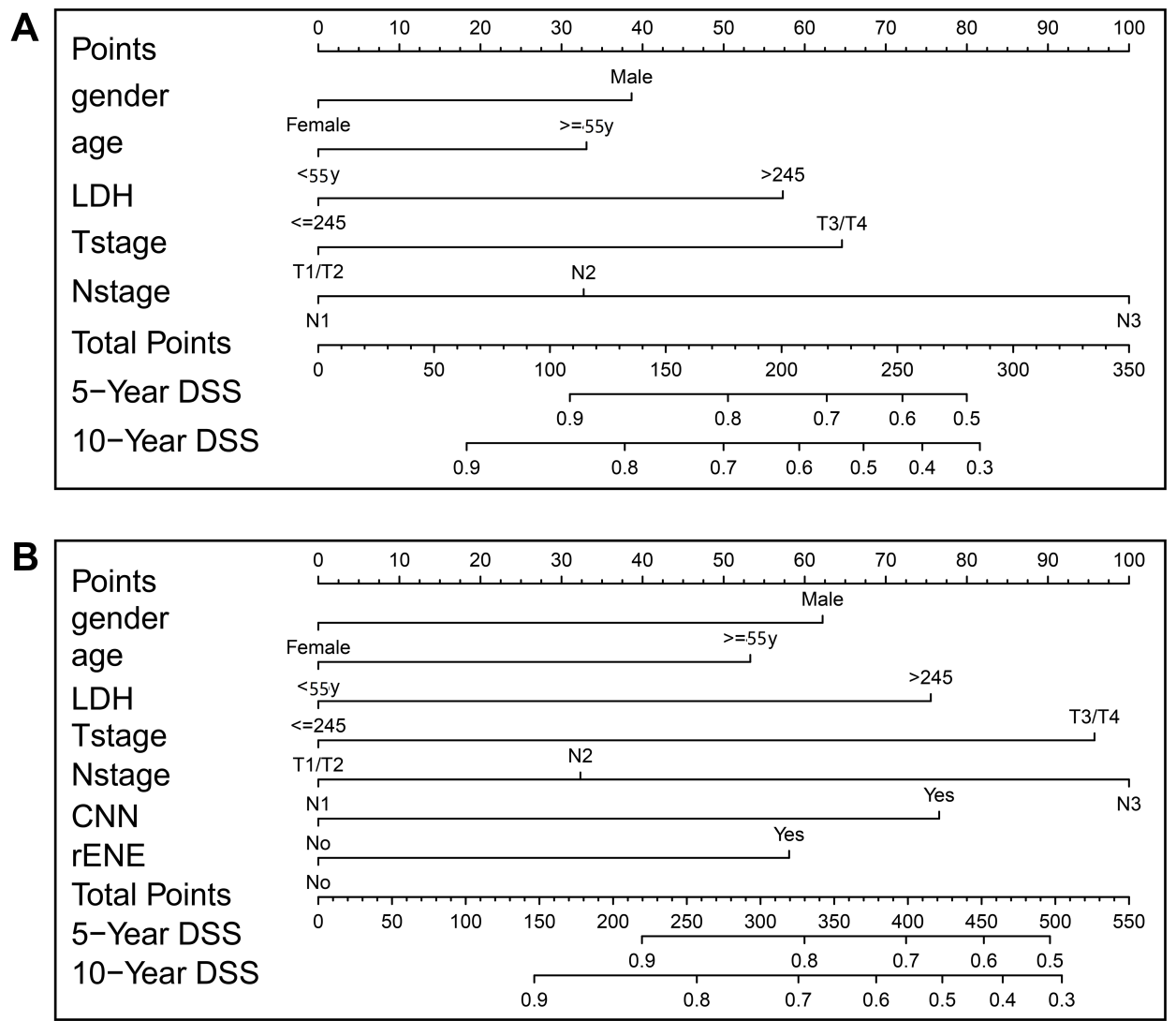

Figure 4 Nomogram (A) (without nodal features) and nomogram (B) (with nodal features) developed for the 5- and I0-year prediction of disease-specific survival (DSS).
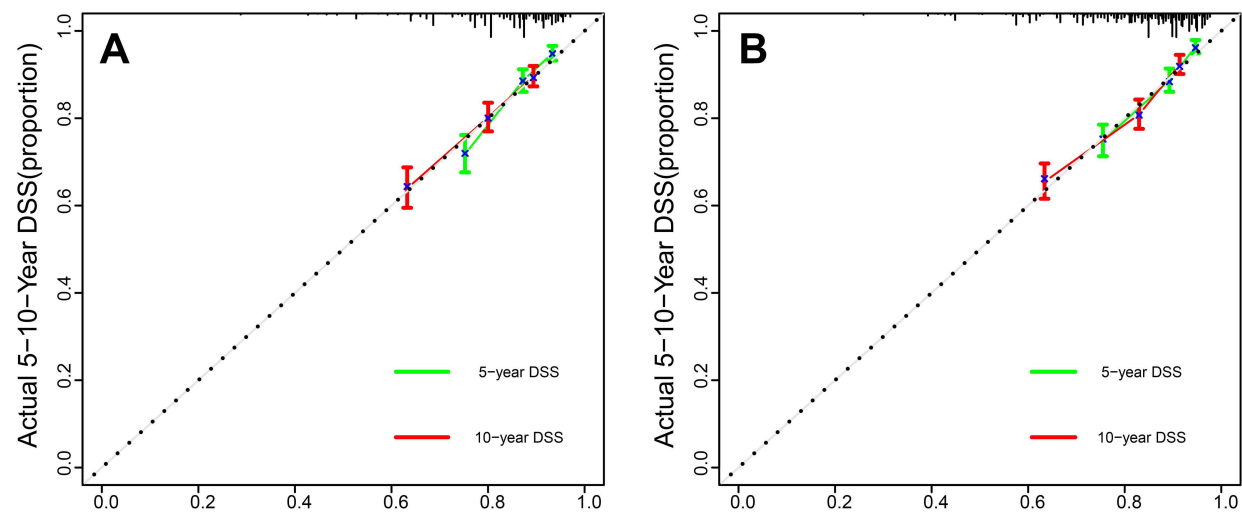

Nomogram-Predicted Probability of $5-10-$ Year DSS

Figure 5 The calibration curve for predicting the 5- and 10-year DSS with nomogram (A) and nomogram (B).

other centers/institutions are warranted for validation of our results.

In conclusion, the nodal features including ENE and $\mathrm{CNN}$ were independent negative prognostic factors for
DSS and DMFS in patients with NPC, and the nomogram that included the nodal features showed better accuracy in predicting survival than the nomogram that excluded the nodal features. 


\section{Data Sharing Statement}

The data supporting the conclusion of this article is available from Yun-Ming Tian and Fei Han.

\section{Funding}

This work was supported by the Medical Scientific Research Foundation of Guangdong Province of China (A2020504).

\section{Disclosure}

Yun-Ming Tian and Lei Zeng shared the first authorship. Li Bai and Fei Han shared the senior authorship. The authors report no conflicts of interest in this work.

\section{References}

1. Yu MC, Yuan JM. Epidemiology of nasopharyngeal carcinoma. Semin Cancer Biol. 2002;12(6):421-429. doi:10.1016/ S1044579X02000858

2. Bray F, Ferlay J, Soerjomataram I, et al. Global cancer statistics 2018: GLOBOCAN estimates of incidence and mortality worldwide for 36 cancers in 185 countries. CA Cancer J Clin. 2018;68 (6):394-424. doi:10.3322/caac. 21492

3. Mao YP, Liang S-B, Liu L-Z, et al. The N staging system in nasopharyngeal carcinoma with radiation therapy oncology group guidelines for lymph node levels based on magnetic resonance imaging. Clin Cancer Res. 2008;14(22):7497-7503. doi:10.1158/1078-0432. CCR-08-0271

4. Ng SH, Chang TC, Ko SF, et al. Nasopharyngeal carcinoma: MRI and CT assessment. Neuroradiol. 1997;39(10):741-746. doi:10.1007/ s002340050499

5. Pan JJ, Ng WT, Zong JF, et al. Proposal for the 8th edition of the AJCC/UICC staging system for nasopharyngeal cancer in the era of intensity-modulated radiotherapy. Cancer. 2016;122(4):546-558. doi:10.1002/cncr.29795

6. Liu JT, Kann BH, De B, et al. Prognostic value of radiographic extracapsular extension in locally advanced head and neck squamous cell cancers. Oral Oncol. 2016;52:52-57. doi:10.1016/j. oraloncology.2015.11.008

7. Myers JN, Greenberg JS, Mo V, Roberts D. Extracapsular spread, A significant predictor of treatment failure in patients with squamous cell carcinoma of the tongue. Cancer. 2001;92(12):3030-3036. doi:10.1002/1097-0142(20011215)92:12<3030::AIDCNCR10148>3.0.CO;2-P

8. Billfalk-Kelly A, Yu E, Su J, et al. Radiologic extranodal extension portends worse outcome in $\mathrm{cN}+$ TNM-8 stage I human papillomavirus-mediated oropharyngeal cancer. Int J Radiat Oncol Biol Phys. 2019;104(5):10176-11027. doi:10.1016/j. ijrobp.2019.03.047

9. Compton C, Hess KR, Halabi S, et al. Risk models for individualized prognosis in the practice of precision oncology. In: AJCC Cancer Staging Manual. $8^{\text {th }}$ ed. Springer International Publishing; 2017:47-52

10. Zhang LL, Li J-X, Zhou G-Q, et al. Influence of cervical node necrosis of different grades on the prognosis of nasopharyngeal carcinoma patients treated with intensity-modulated radiotherapy. $J$ Cancer. 2017;8(6):959-966. doi:10.7150/jca.17998

11. Ai QY, King AD, Poon DMC, et al. Extranodal extension isa criterion for poor outcome in patients with metastatic nodes from cancer of thenasopharynx. Oral Oncol. 2019;88:124-130. doi:10.1016/j. oraloncology.2018.11.007
12. Lu T, Hu Y, Xiao Y, et al. Prognostic value of radiologic extranodal extension and its potential role in future $\mathrm{N}$ classification for nasopharyngeal carcinoma. Oral Oncol. 2019;99:104438. doi:10.1016/j. oraloncology.2019.09.030

13. Liu Y, Chen S, Dong A, et al. Nodal grouping in nasopharyngeal carcinoma: prognostic significance, $\mathrm{N}$ classification, and a marker for the identification of candidates for induction chemotherapy. Eur Radiol. 2020;30(4):2115-2124. doi:10.1007/s00330-019-06537-6

14. Brekel MW, Stel HV, Castelijns JA, et al. Cervical lymph node metastasis: assessment of radiologic criteria. Radiology. 1990;177 (2):379-384. doi:10.1148/radiology.177.2.2217772

15. Som PM. Update on imaging metastatic cervical lymph nodes: criteria and differential diagnosis. Am J Roentgenol. 1992;158 (5):961-969. doi:10.2214/ajr.158.5.1566697

16. Tian YM, Liu M-Z, Zeng L, et al. Long-term outcome and pattern of failure for patients with nasopharyngeal carcinoma treated with intensity-modulated radiotherapy. Head Neck. 2019;41 (5):1246-1252. doi:10.1002/hed.25545

17. Harrell FE Jr, Lee KL, Mark DB. Multivariable prognostic models: issues in developing models, evaluating assumptions and adequacy, and measuring and reducing errors. Stat Med. 1996;15(4):361-387. doi:10.1002/(SICI)1097-0258(19960229)15:4<361::AID-SIM168>3. $0 . \mathrm{CO} ; 2-4$

18. Frank EHJ Harrell Miscellaneous. R Package version 3.6.1.; 2019. Available from: http://CRAN.Rproject.org/package.Hmisc. Accessed June 9, 2021.

19. Au KH, Ngan RKC, Ng AWY, et al. Treatment outcomes of nasopharyngeal carcinoma in modern era after intensity modulated radiotherapy (IMRT) in Hong Kong: a report of 3328 patients (HKNPCSG 1301 study. Oral Oncol. 2018;77:16-21. doi:10.1016/j. oraloncology.2017.12.004

20. Lee AW, Poon YF, Foo W, et al. Retrospective analysis of 5037 patients with nasopharyngeal carcinoma treated during 1976-1985: overall survival and patterns of failure [J]. Int J Radiat Oncol Biol Phys. 1992;23(2):261-270. doi:10.1016/0360-3016(92)90740-9

21. Pereira ER, Kedrin D, Seano G, et al. Lymph node metastases can invade local blood vessels, exit the node, and colonize distant organs in mice. Science. 2018;359(6382):1403-1407. doi:10.1126/science.aal3622

22. Kimura Y, Sumi M, Sakihama N, Tanaka F, Takahashi H, Nakamura T. MR imaging criteria for the prediction of extranodal spread of metastatic cancer in the neck. AJNR Am J Neuroradiol. 2008;29(7):1355-1359. doi:10.3174/ajnr.A1088

23. Ljungkvist AS, Bussink J, Rijken PFJW, et al. Vascular architecture, hypoxia, and proliferation in first-generation xenografts of human head-and-neck squamous cell carcinomas. Int J Radiat Oncol Biol Phys. 2002;54(1):215-228. doi:10.1016/S0360-3016(02)02938-3

24. Zoumalan RA, Kleinberger AJ, Morris LGT, et al. Lymph node central necrosis on computed tomography as predictor of extracapsular spread in metastatic head and neck squamous cell carcinoma: pilot study. J Laryngol Otol. 2010;124(12):1284-1288. doi:10.1017/S0022215110001453

25. Lan M, Huang Y, Chen C-Y, et al. Prognostic value of cervical nodal necrosis in nasopharyngeal carcinoma: analysis of 1800 patients with positive cervical nodal metastasis at MR imaging. Radiology. 2015;276(2):536-544. doi:10.1148/radiol.15141251

26. Tang LQ, Li CF, Li J, et al. Establishment and validation of prognostic nomograms for endemic nasopharyngeal carcinoma. $J$ Natl Cancer Inst. 2015;14:108.

27. Zeng L, Guo P, Li J-G, et al. Prognostic score models for survival of nasopharyngeal carcinoma patients treated with intensity-modulated radiotherapy and chemotherapy. Oncotarget. 2015;6 (36):39373-39383. doi:10.18632/oncotarget.5781

28. Sun Y, Li W-F, Chen N-Y, et al. Induction chemotherapy plus concurrent chemoradiotherapy versus concurrent chemoradiotherapy alone in locoregionally advanced nasopharyngeal carcinoma: a Phase 3, multicentre, randomised controlled trial. Lancet Oncol. 2016;17(11):1509-1520. doi:10.1016/S1470-2045(16)30410-7 


\section{Publish your work in this journal}

Cancer Management and Research is an international, peer-reviewed open access journal focusing on cancer research and the optimal use of preventative and integrated treatment interventions to achieve improved outcomes, enhanced survival and quality of life for the cancer patient.
The manuscript management system is completely online and includes a very quick and fair peer-review system, which is all easy to use. Visit http://www.dovepress.com/testimonials.php to read real quotes from published authors. 